\title{
Asynchronous Non-Invasive Brain-Actuated Control of an Intelligent Wheelchair
}

\author{
J. del R. Millán, F. Galán, D. Vanhooydonck, E. Lew, J. Philips and M. Nuttin
}

\begin{abstract}
In this paper we present further results of our asynchronous and non-invasive BMI for the continuous control of an intelligent wheelchair. Three subjects participated in two experiments where they steered the wheelchair spontaneously, without any external cue. To do so the users learn to voluntary modulate EEG oscillatory rhythms by executing three mental tasks (i.e., mental imagery) that are associated to different steering commands. Importantly, we implement shared control techniques between the BMI and the intelligent wheelchair to assist the subject in the driving task. The results show that the three subjects could achieve a significant level of mental control, even if far from optimal, to drive an intelligent wheelchair.
\end{abstract}

\section{INTRODUCTION}

The idea of moving robots or prosthetic devices not by manual control but by mere "thinking" - that is, by human brain activity - has fascinated researchers for the past 40 years. But only recently have experiments shown the feasibility of using brain-machine interfaces (BMI) for controlling and interacting with robots and wheelchairs [1], [2], [3], [4], [5], [6], [7].

A BMI monitors the user's brain activity and translates their intentions into actions — such as driving a wheelchair or selecting a letter from a virtual keyboard - without using activity of any muscle or peripheral nerve. The central tenet of a BMI is the capability to distinguish different patterns of brain activity, each being associated to a particular intention or mental task. Hence adaptation is a key component of a BMI because users must learn to modulate their brainwaves so as generate distinct brain patterns. In some cases, user training is complemented with machine learning techniques to discover the individual brain patterns characterizing the mental tasks executed by the user.

What kind of brain signals can directly control robots? Electrical activity is the natural candidate because of its excellent time resolution. Most of the hope for brain-controlled robots comes from invasive approaches that provide detailed, single neuron activity recorded from microelectrodes implanted in the brain [1], [6]. The motivation for these invasive approaches is the broad evidence that ensembles of neurons in the brain's motor system - primary motor, premotor, and posterior parietal cortex - encode the parameters of

This work was supported in part by the European IST Programme FET Project FP6-003758 (MAIA). JdRM, FG and EL were with Idiap Research Institute, Martigny (Switzerland), when this work was carried out

J. del R. Millán and E. Lew are with the Ecole Polytechnique Fédérale de Lausanne (EPFL), Switzerland. jose.millan@epfl. ch

F. Galán is with the Albert-Ludwigs University, Freiburg, Germany.

D. Vanhooydonck, J. Philips and M. Nuttin are with the Katholieke Universiteit Leuven (KUL), Belgium. limb movements in a distributed, redundant way involving thousands of neurons over several brain cortical areas.

For humans, however, non-invasive approaches avoid health risks and associated ethical concerns. Most noninvasive BMI use electroencephalogram (EEG) signals - the electrical brain activity recorded from electrodes placed on the scalp. The main source of the EEG is the synchronous activity of thousands of cortical neurons. Thus, EEG signals suffer from a reduced spatial resolution and increased noise when measurements are taken on the scalp. Consequently, current EEG-based brain-actuated devices are limited by a low information transfer rate and are considered too slow for controlling rapid and complex sequences of robot movements. Recently, however, we have shown for the first time that online analysis of EEG signals, if used in combination with advanced robotics and machine learning techniques, is sufficient for humans to continuously control a mobile robot [2] and a wheelchair [4], [5].

For brain-controlled robots, in contrast to augmented communication through BMI, fast decision-making is critical. In our view, continuous mental control of robots requires the analysis of EEG components associated with spontaneous, intentional mental activity. An alternative is to exploit evoked potentials — the immediate automatic responses of the brain to external stimuli. Examples of evoked potentials include P300 and SSVEP (steady-state visual evoked potentials). In principle, evoked potentials are easy to detect with scalp electrodes. The necessity of external stimulation does, however, restrict the applicability of evoked potentials to a limited range of tasks. As in driving a car, the subjects' attention must focus on driving and not on external stimuli.

But voluntary mental control is not enough for steering a wheelchair or a prosthesis. These tasks require subjects to also make self-paced decisions. In such asynchronous protocols, the subject can deliver a mental command at any moment without waiting for external cues [8], [2], [9]. This contrasts with synchronous interaction, where the EEG is time-locked to externally paced cues. Only asynchronous BMIs allow subjects to send the appropriate mental command at the right time to make the wheelchair turn and cross the desired doorway while it is moving continuously.

Despite that asynchronous spontaneous BMIs seem to be the most natural and suitable alternative, there are a few examples of synchronous evoked BMIs for the control of wheelchairs [3], [7]. Both systems are based on P300, a potential evoked by an awaited infrequent stimulus. To evoke the P300, the system flashes the possible target destinations several times in a random order. The subject's choice is the 


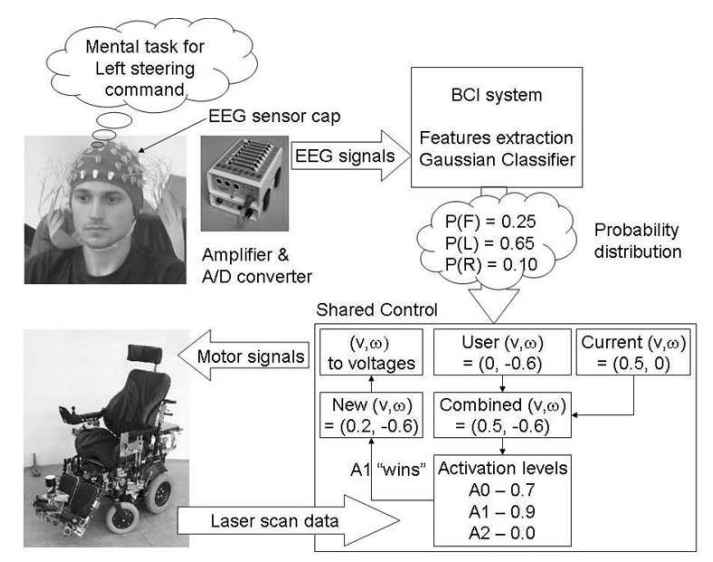

Fig. 1. Diagram of how a mental steering command is integrated in the shared control system and eventually converted into motor signals. In this example, obstacle avoidance is the winning behavior and adjusts the velocities to prevent collision.

stimulus that elicits the largest P300. Then, the intelligent wheelchair reaches the selected target autonomously. Once there, it stops and the subject can select another destination - a process that takes around 10 seconds.

In this paper we present further results of our asynchronous and non-invasive BMI for the continuous control of an intelligent wheelchair. As mentioned previously, users steer the wheelchair spontaneously, without any external cue. To do so the users learn to voluntary modulate EEG oscillatory rhythms by executing three mental tasks (i.e., mental imagery) that are associated to different steering commands. Importantly, we implement shared control techniques between the BMI and the intelligent wheelchair to assist the subject in the driving task [10], [11] (see Fig. 1). Here we describe two experiments on increasing difficulty where three subjects demonstrated a significant level of mental control of an intelligent wheelchair.

\section{METHODS}

\section{A. EEG Protocol}

Data was recorded with a portable Biosemi acquisition system using 64 channels sampled at $512 \mathrm{~Hz}$ and high-pass filtered at $1 \mathrm{~Hz}$. Then, the signal was spatially filtered using a common average reference (CAR) before estimating the power spectral density (PSD) in the band 8-46 Hz with $2 \mathrm{~Hz}$ resolution over the last 1 second. The PSD was estimated every $62.5 \mathrm{~ms}$ (i.e., 16 times per second) using the Welch method with 5 overlapped (25\%) Hanning windows of 500 ms. Thus, an EEG sample was a 1344-dimensional vector (64 channels times 21 frequency components).

Obviously, not all these 1344 features are used as control signals. [4] describes the algorithms to estimate the relevance of the features for discriminating the mental commands and the procedure to select the most stable discriminant features that are sent to the statistical classifier embedded in the BMI. This classifier processes each of the EEG samples and the BMI combines 8 consecutive responses to deliver a mental command every 0.5 seconds.
The three subjects participated in 20 calibration sessions utilized to extract subject specific stable discriminant EEG features and build a statistical Gaussian classifier (see [2] for details) for each subject. In these sessions, the subjects sat in a chair looking at a fixation point placed in the center of a monitor. The display is a simulated wheelchair in a first person view. The subjects were asked to execute the three mental tasks in a counterbalanced order informing the operator when they started executing the task. Each calibration session was integrated by 6 trials each, 2 trials per class. Each trial lasted for 7 seconds but only the last 6 were utilized in the analysis to avoid preparation periods where the subjects were not yet engaged in the execution of the mental task. During these sessions the subjects did not received any feedback, so the monitor display is static.

To drive the wheelchair, subjects 1 and 2 utilized the three mental commands: imagination of a left hand movement, words associations and relaxation. These mental commands were respectively associated with the three wheelchair steering behaviors: turn left, turn right and move forward. Subject 3 utilized different mental commands: words associations, arithmetic operations and relaxation, associated with the aforementioned steering behaviors, respectively.

\section{B. Shared Control}

The concept of shared control is used in robotics when both an intelligent system and a human operator are in control of a system. In the case of an intelligent wheelchair, the task of the system is to provide navigational assistance to users, when they are not able to execute certain manoeuvres safely and independently. In other words, when users need no navigational assistance to achieve their plan or intention, they will be granted full control over the wheelchair; otherwise, their mental commands will be modified by the system appropriately. A key aspect of shared control is that a successful human-robot cooperation requires a good twoway communication - the behavior of the robot should be intuitive to the user and the robot should unambiguously understand the user's commands.

There exist two reasons why shared control is beneficial for mental control of a wheelchair by means a 3-class BMI. Firstly, as shown in Fig. 1, there are "only" 3 possible steering mental commands: Forward, Left, and Right. So, fine manoeuvring demands some assistance by the system. Secondly, the mental commands (the outputs of the BMI) are not always perfect. In case of such errors, the system has to provide extra navigational safety.

Fig. 1 shows the shared control approach we have adopted. It is agent-based, where several types of assistive behaviors are implemented as different agents. The inputs to the shared control module are the sensory readings of the wheelchair (a laser range finder) and the outputs of the BMI (a probability distribution over the three possible steering mental commands generated at $2 \mathrm{~Hz}$ ). The mental command with the highest probability is converted to a motor signal (translational and rotational velocities $v$ and $\omega$ ). This new motor signal is then combined with the current wheelchair 


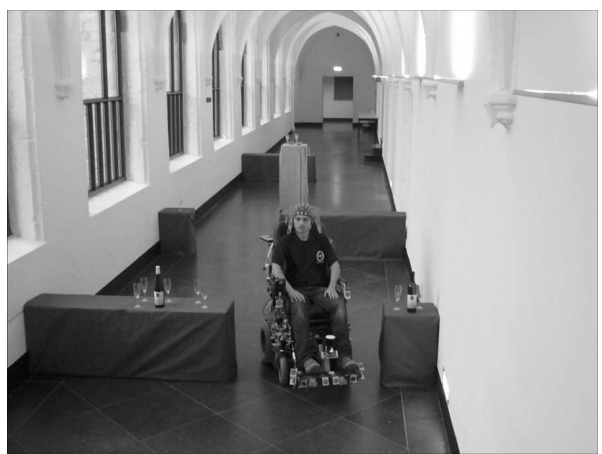

Fig. 2. Indoor environment utilized in task 1 . The starting point was the far extreme in the picture. The subjects were asked to mentally drive the wheelchair to the other extreme (lower part of the picture).

velocity, in order to generate a smoother driving behavior. Finally, shared control uses the resulting motor signal and the sensory readings to select the winning assistive behavior.

\section{Experimental Tasks and Analysis}

1) Task 1. Slalom: Two healthy subjects were asked to mentally drive a wheelchair in an indoor environment including human activity. Subject 1 had previous experience driving in simulated environments but no experience driving the real wheelchair. Subject 2 had previous experience driving in simulated and real conditions ( 3 days with the real wheelchair). The task was to drive the wheelchair along a corridor up to the end while avoiding obstacles (see Fig. 2). The dimensions of the experimental arena were 19 by 3.5 meters. Subject 1 performed 11 trials and subject 2 did 14 trials. The objective of this experiment was twofold, namely to demonstrate for the first time the possibility of continuously driving a complex robotics device such as a wheelchair in a natural environment using a BMI and to assess the amount of assistance required during the driving task. To do so, the percentage of corrective actions by obstacle avoidance were analyzed over all the trials.

2) Task 2. Docking: Both subjects that participated in task 1 and a third one without any driving experience (simulated or real) were asked to mentally drive the wheelchair to reach 3 target locations while avoiding obstacles (see Fig. 3). Reaching a target is a more complex task than simply navigating, as in the previous task. This experiment is more challenging in a second respect, namely subjects cannot manoeuver back the wheelchair if they overshot the target by more than 2 meters, thus missing the correct turn. If this is the case, the trial was considered a failure.

The motivation for this experiment is to assess how well naive (or almost naive) subjects can mentally drive the wheelchair along "almost" optimal trajectories. To measure the performance of our brain-actuated wheelchair we have compared the final position of the wheelchair with the end point of the desired trajectory. In particular, we have calculated the percentage of reached targets as a function of the distance between the final wheelchair position and the target at each trial. Furthermore, to assess the degree of

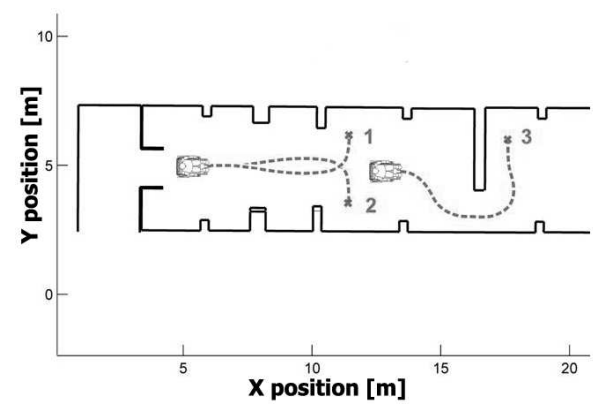

Fig. 3. Indoor environment utilized in task 2. The subjects were asked to drive the wheelchair to targets 1,2 and 3. The figure also depicts the initial positions and ideal trajectories for each target.

mental control achieved by the subjects, their performances were compared with that of a random BMI utilized as a baseline -i.e., the wheelchair was driven by such a random BMI. Each subject, as well as the random BMI, participated in 30 randomized trials (10 trials per target).

\section{RESULTS}

\section{A. Experimental Task 1. Slalom}

Subjects performed all the trials in a row, without long resting times between trials. To perform a trial, subjects needed to operate the BMI for a rather long time - the total time to navigate along the corridor was in between 250 and 350 seconds, where subjects were delivering mental commands at $2 \mathrm{~Hz}$. So, subjects reported that they suffered from fatigue at several points during the experiments, but we didn't stop experiments to allow them to recover. Both subjects achieved the task of driving the wheelchair to the end of the corridor while avoiding obstacles. Significantly, they could do so even at the first trial. As previous experiments with a simulated wheelchair showed, however, performance improved with experience [11].

But, as any robotician knows, it is always possible to program an intelligent wheelchair to navigate in a prespecified environment autonomously. So, the question arises of how much degree of mental control the subjects actually have. To answer this question, Fig. 4 shows the percentage of corrective actions executed by the activation of the obstacle avoidance behavior of the shared control module over all the trials for the two subjects. At all other times, the user was in total control of the wheelchair. As the figure shows, users needed a rather low level of assistance that was variable along the trials and depended on the context as well as the level of fatigue and concentration of the subjects.

\section{B. Experimental Task 2. Docking}

Fig. 5 shows the percentage of targets reached by each subject and the random BMI as a function of the distance between the final wheelchair position and the target at each trial. The results reflect the importance of previous experience to successfully drive the wheelchair. Subject 2, who had previous driving experience with both the simulated and the real wheelchair, brought it closer to the targets. On 

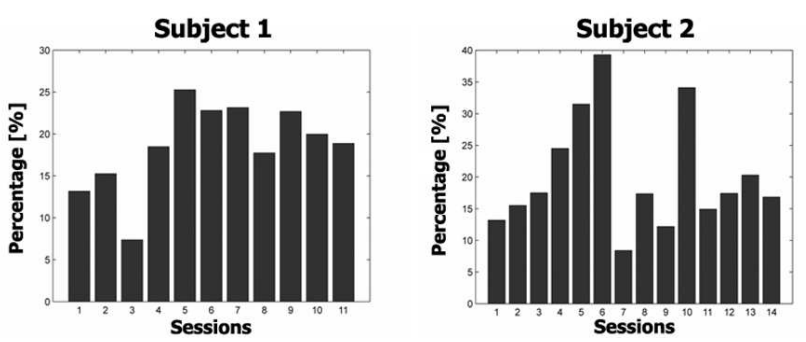

Fig. 4. Percentage of corrective actions provided by the obstacle avoidance behavior of the shared control for the two subjects over all the trials.

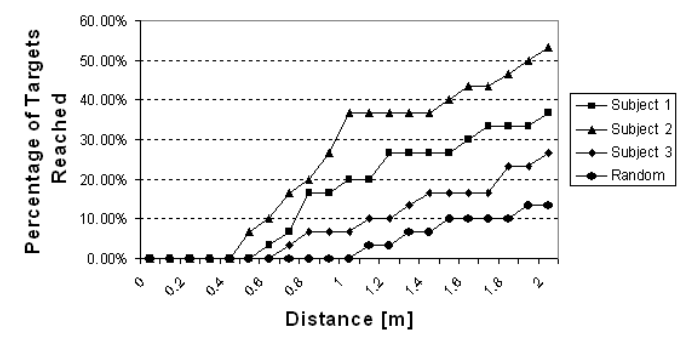

Fig. 5. Percentage of reached targets by each subject and the random BMI as a function of the distance between the final position and the target.

the contrary, subject 3, who did not have any previous driving experience, had more difficulties to place the wheelchair close to the targets. Subject 1 , who had only previous experience in simulation, achieved an intermediate performance.

Despite the different driving performances among subjects, the three of them showed a significant degree of mental control of the wheelchair, which requires rather fast and accurate decisions. For instance, to drive the wheelchair to target 3 , the most difficult one, the subject needs to pass through the narrow passage in the opposite direction, right, and then immediately make a sharp turn to the left. Interestingly, subjects missed quite a few times targets 1 and 2 because they tried to reach them following a straight line and the collision avoidance behavior (for details see [10], [11]) pushed the wheelchair away from the target. As shown in fig. 3, the optimal trajectory is not straight, but the subjects needed some time to learn appropriate driving strategies compatible with the behavior of the intelligent wheelchair.

To measure the degree of mental control exhibited by the subjects, and to show the complexity of the task, we run an experiment where the wheelchair was driven by a random BMI (i.e., the mental steering command - left, right, or forward-was selected randomly every 0.5 seconds). The performance of such a random BMI was such that it never brought the wheelchair closer than 1 meter from the target whereas subjects 1, 2 and 3 did it in $20 \%, 37 \%$ and $7 \%$ of the trials, respectively. The subjects' level of mental control is even higher when we consider the percentage of trials where the wheelchair was driven closer than 2 meters from the target. In this case, subjects 1, 2 and 3 achieved the task in $37 \%, 53 \%$ and $27 \%$ of the trials, whereas the random BMI did it only in $13 \%$ of the trials.

\section{CONCLUSiOnS}

Our experimental results show that subjects can operate our asynchronous non-invasive BMI to control a wheelchair, task that requires rather fast and accurate decisions. It is worth noting that, by virtue of shared control, tasks become possible to achieve even at the first time or day that they are performed. However, the performances seem to be lower than the obtained with the simulated version of the wheelchair [4]. Moreover, subjects 1 and 2, who had previous experience with the simulated wheelchair, report that it is more difficult to drive the real wheelchair because of its more complex behavior. Nevertheless, the performance of the subjects, even the naive subject, is significantly better than a random BMI. Also, as the first experiments shows, the level of assistance provided by the shared control module is rather modest. Altogether, the results prove that the intelligent wheelchair cannot achieve the task by itself, but requires appropriate mental commands delivered by the subject at the right times.

In summary, results show that subjects can rapidly achieve a significant level of mental control, even if far from optimal, to drive an intelligent wheelchair, thus demonstrating the feasibility of continuously controlling complex robotics devices using an asynchronous and non-invasive BMI.

\section{REFERENCES}

[1] J.M. Carmena, M.A. Lebedev, R.E. Crist, J.E. ODoherty, D.M. Santucci, D.F. Dimitrov, P.G. Patil, C.S. Henriquez, and M.A.L. Nicolelis, "Learning to control a brain-machine interface for reaching and grasping by primates," PLoS Biology, vol. 1, pp. 193-208, 2003.

[2] J. del R. Millán, F. Renkens, J. Mouriño, and W. Gerstner, "Noninvasive brain-actuated control of a mobile robot by human EEG," IEEE Trans. Biomed. Eng., vol. 51, pp. 1026-1033, 2004.

[3] B. Rebsamen, E. Burdet, C.L. Teo, Q. Zeng, C. Guan, M. Ang, and C. Laugier, "A brain control wheelchair with a p300 based bci and a path following controller," in Proc. 1st IEEE/RAS-EMBS Int. Conf. Biomedical Robotics and Biomechatronics, Pisa, Italy, 2006.

[4] F. Galán, M. Nuttin, E. Lew, P.W. Ferrez, G. Vanacker, J. Philips, and J. del R. Millán, "A brain-actuated wheelchair: Asynchronous and noninvasive brain-computer interfaces for continuous control of robots," Clin. Neurophysiol., vol. 119, pp. 2159-2169, 2008.

[5] F. Galán, M. Nuttin, D. Vanhooydonck, E. Lew, P.W. Ferrez, J. Philips, and J. del R. Millán, "Continuous brain-actuated control of an intelligent wheelchair by human EEG," in Proc. 4th Int. BrainComputer Interface Workshop, Graz, Austria, 2008.

[6] M. Velliste, S. Perel, M.C. Spalding, A.S. Whitford, and A.B. Schwartz, "Cortical control of a prosthetic arm for self-feeding," Nature, vol. 453, pp. 1098-1101, 2008.

[7] I. Iturrate, J. Antelis, and J. Minguez, "Synchronous EEG brainactuated wheelchair with automated navigation," in Proc. 2009 IEEE Int. Conf. Robotics Automation, Kobe, Japan, 2009.

[8] G.E. Birch, Z. Bozorgzadeh, and S.G. Mason, "Initial on-line evaluation of the LF-ASD brain-computer interface with able-bodied and spinal-cord subjects using imagined voluntary motor potentials," IEEE Trans. Neural Sys. Rehab. Eng., vol. 10, pp. 219-224, 2002.

[9] R. Scherer, G.R. Müller, C. Neuper, B. Grainmann, and G. Pfurtscheller, "An asynchronous controlled EEG-based virtual keyboard: Improvement of the spelling rate," IEEE Trans. Biomed. Eng., vol. 51, pp. 979-984, 2004.

[10] J. Philips, J. del R. Millán, G. Vanacker, E. Lew, F. Galán, P.W. Ferrez, H. Van Brussel, and M. Nuttin, "Adaptive shared control of a brainactuated simulated wheelchair," In Proc. 10th Intl. Conf. Rehabilitation Robotics, 2007.

[11] G. Vanacker, J. del R. Millán, E. Lew, P.W. Ferrez, F. Galán, J. Philips, H. Van Brussel, and M. Nuttin, "Context-based filtering for assisted brain-actuated wheelchair driving," Computational Intelligence and Neuroscience, 2007, Article ID 25130, 2007. 